\title{
Simulation and measurement of total ionizing dose radiation induced image lag increase in pinned photodiode CMOS image sensors
}

\author{
Jing Liu ${ }^{\mathrm{a}, \mathrm{b}}$, Wei Chen ${ }^{\mathrm{b}, *}$, Zujun Wang ${ }^{\mathrm{b}, *}$, Yuanyuan Xue ${ }^{\mathrm{b}}$, Zhibin Yao ${ }^{\mathrm{b}}$, Baoping He ${ }^{\mathrm{b}}$, \\ Wuying Ma ${ }^{\mathrm{b}}$, Junshan Jin ${ }^{\mathrm{b}}$, Jiangkun Sheng ${ }^{\mathrm{b}}$, Guantao Dong ${ }^{\mathrm{b}}$. \\ a School of Materials Science and Engineering, Xiangtan University, Hunan, China \\ $b$ State Key Laboratory of Intense Pulsed Irradiation Simulation and Effect, Northwest Institute of Nuclear Technology, \\ P.O.Box 69-10, Xi'an, CHN
}

\begin{abstract}
This paper presents an investigation of total ionizing dose (TID) induced image lag sources in pinned photodiodes (PPD) CMOS image sensors based on radiation experiments and TCAD simulation. The radiation experiments have been carried out at the Cobalt -60 gamma-ray source. The experimental results show the image lag degradation is more and more serious with increasing TID. Combining with the TCAD simulation results, we can confirm that the junction of PPD and transfer gate (TG) is an important region forming image lag during irradiation. These simulations demonstrate that TID can generate a potential pocket leading to incomplete transfer.
\end{abstract}

Keywords - CMOS image sensors; total ionizing dose; image lag; pinned photodiodes

\section{Introduction}

Pinned photodiode (PPD) CMOS Image Sensors (CISs), also called 4T PPD CISs, are the most potential photodetector, which exhibits lower noise, lower dark current, lower power consumption, and higher reliability compared with conventional CIS based on 3T PD (photodiode) [1-3]. It is widely used in some high performance imaging areas, such as nuclear industry, medical imaging, and space mission [4-5]. Image lag is one of the most important parameters of the CIS performance [6]. So far, very few reports have been published on image lag induced by TID in 4T PPD CIS [9-10]. The TID induced degradation mechanism proposed in these previous studies has yet to be confirmed by simulation.

The aim of this work is to confirm one of the origins of the TID effects induced the image lag. Firstly, the degradation of the image lag was observed by radiation experiments. Then, by using TCAD simulation, we can confirm the TID radiation sensitivity related to the image lag problem. Finally, by analyzing the results of experiment and simulation, the degradation mechanism of image lag is discussed.

\section{Experimental Details}

The device is a $2048 \times 20484$ T-PPD pixel array (see Fig. 1 for a simplified 4T PPD cross-section), manufactured in a commercial $0.18 \mu \mathrm{m}$ CMOS process. The $\gamma$ photons produced by a Cobalt-60 gamma-ray source (at the Northwest Institute of Nuclear Technology, Xi'an, CHN) and the dose rate is calibrated by PTW-UNIDOS. The CISs are measured at accumulated doses of $50,100,150,200 \mathrm{krad}(\mathrm{Si})$ during irradiation test, and the dose rate is about $10 \mathrm{rad}(\mathrm{Si}) / \mathrm{s}$. The biasing condition is grounding the devices during irradiation at room temperature.

The image lag test method used in this paper is as follows: First, a pulsed light source is used; find an exposure time until at least $50 \%$ of saturation. Then, at this exposure time, 10 full scale images are grabbed. Among them, the fifth image is grabbed during the pulsed light source is turned on; the other images are grabbed in a completely dark condition. Finally, the image lag is calculated by:

$$
\text { image lag }=\frac{\left|\mu_{6, \text { dark }}-\bar{\mu}_{\text {dark }}\right|}{\left|\mu_{5, \text { pulse }}-\bar{\mu}_{\text {dark }}\right|} \times 100 \%
$$

\footnotetext{
${ }^{*}$ Corresponding author. Tel.: +86-29-84765134; Fax: +86-29-83366333

E-mail address: chenwei@nint.ac.cn (Wei Chen); wangzujun@nint.ac.cn (Zujun Wang)
} 
where $\mu_{6, \text { dark }}$ the mean digital signal of the sixth image is in a completely dark condition, $\mu_{5, \text { pulse }}$ is the mean digital signal of the fifth image during the pulse light source is turned on. $\bar{\mu}_{\text {dark }}$ represents the average gray value of the remaining eight images.

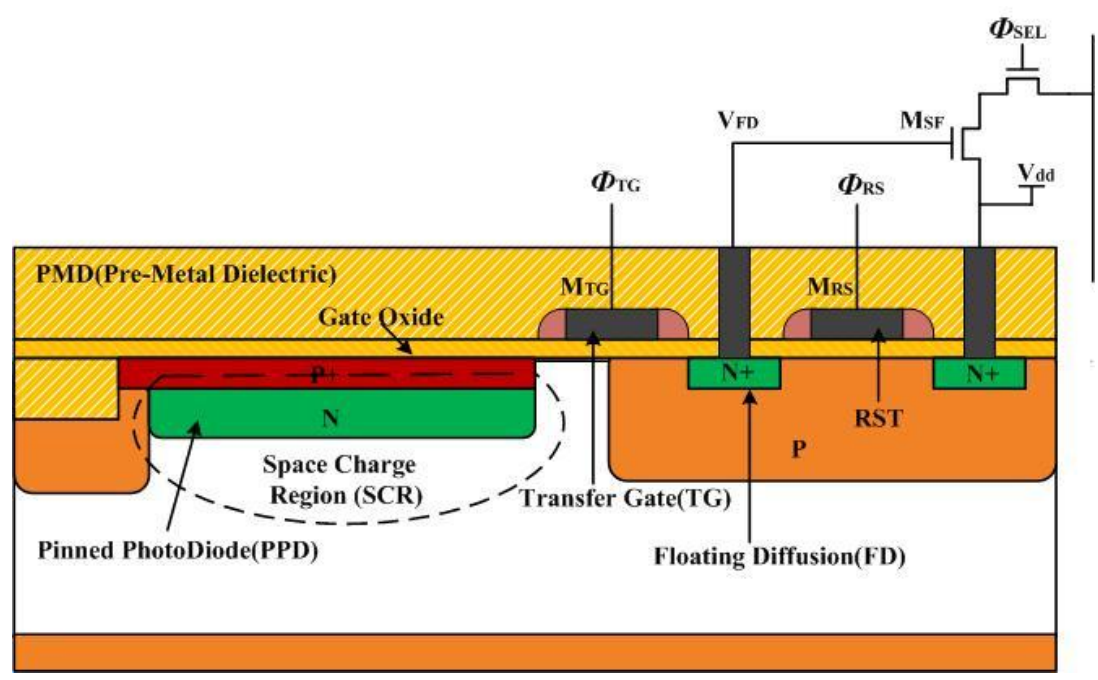

Fig. 1 Cross sectional view of a 4T PPD pixel, inspired from [8].

\section{Experimental results}

The image lag refers to the phenomenon that the residual signal charge is reproduced at the time of the next image formation, due to incomplete transfer of the signal charge. As the 4T PPD CIS's operating voltage is low (generally 2.5 3.3 V), the 4T PPD CIS are more likely to cause an image lag problem [7-8].

Fig. 2 shows the image lag as a function of TID at the dose rate of $10 \mathrm{rad}(\mathrm{Si}) / \mathrm{s}$. From Fig. 2, one can see that the image lag increases with increasing TID. When the TID is lower than $100 \mathrm{krad}(\mathrm{Si})$, the image lags increase slightly. However, when the TID is higher than $100 \mathrm{krad}(\mathrm{Si})$, the image lags increase remarkably. When the accumulated dose reaches $200 \mathrm{krad}(\mathrm{Si})$, the image lags are four times as large as that of pre-irradiation. Generally speaking, we believe that one of the image lag origins is long transit time from the PPD to the float diffusion (FD) node. When there is a potential barrier or pocket between the photodiode and the FD node, the time needed to achieve a full transfer increases with increasing TID.

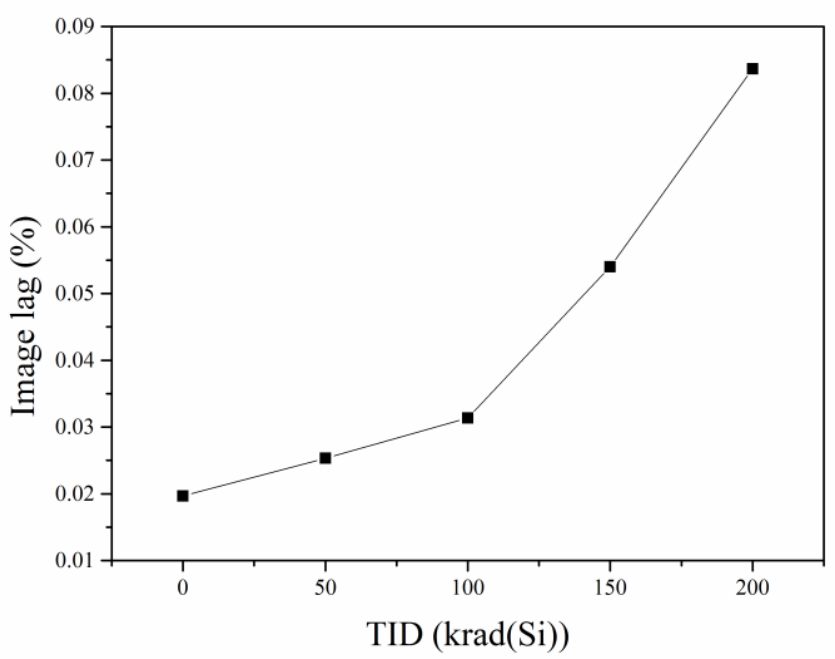

Fig. 2 The curve of the image lags with TID increase.

\section{Simulation results}


It is well known that the main origin of image lag in 4T PPD CIS is a potential barrier or pocket between the PPD region and the TG region. Radiation induced positive charges trapped in the dielectrics around this overlap region (e.g., the Pre-Metal Dielectric (PMD) and the spacers) lead to an additional electric filed that trends to lower potential barriers and increase the depth of potential pockets [9-10]. In this paper, the TCAD simulation model was used to qualitatively find the causes of image lag degradation induced by TID.

In this TCAD simulation model, the following models were took into consideration: the defect model about trapped positive charge, the radiation model, optical generation model, Shockley-Read-Hall (SRH) recombination, mobility model and so on. Several free parameters involved in this radiation model include $\mathrm{g}, \mathrm{E}_{0}, \mathrm{E}_{1}$ and $\mathrm{m}$, where $\mathrm{E}_{1}$ equals $0.65 \mathrm{MV} / \mathrm{cm}$; the other parameters are default values [13]. The biasing condition is grounding during irradiation by TCAD at room temperature. Fig. 3 shows the element doping profiles of pixel unit by TCAD simulation. Fig. 4 shows that the charge transfers from the PPD region to the FD node when the transfer gate (TG) is turned on by TCAD simulation.

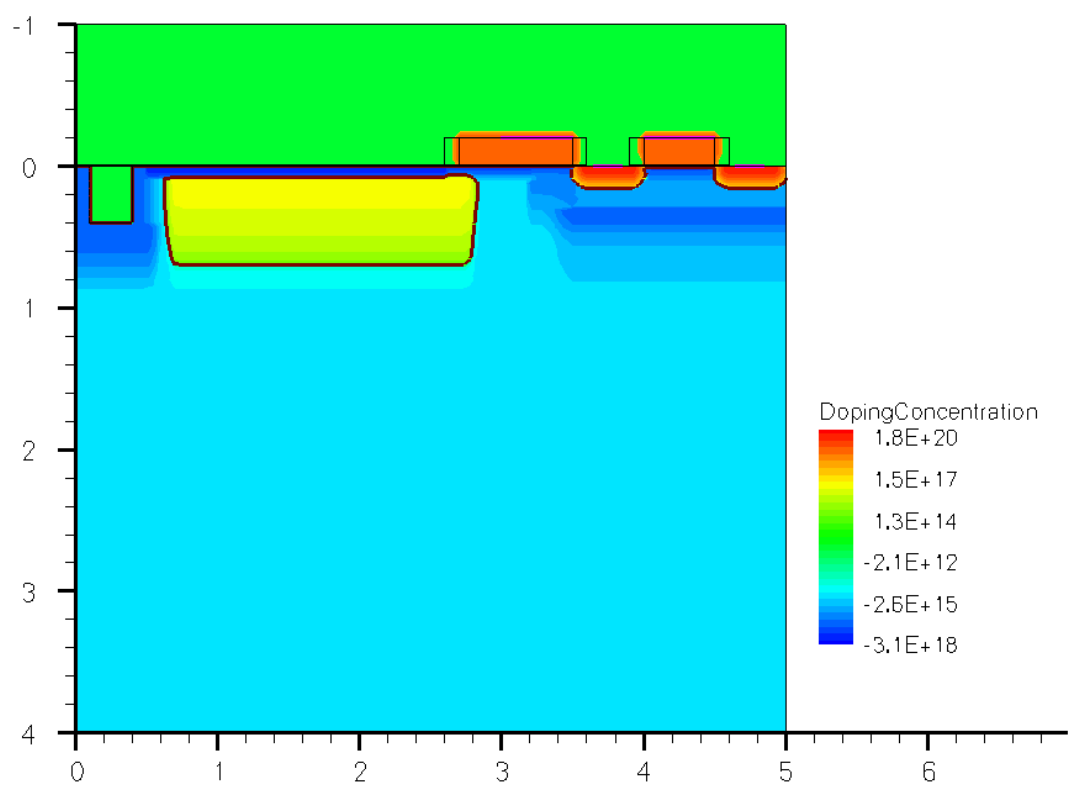

Fig.3 The element doping profiles of the pixel unit.

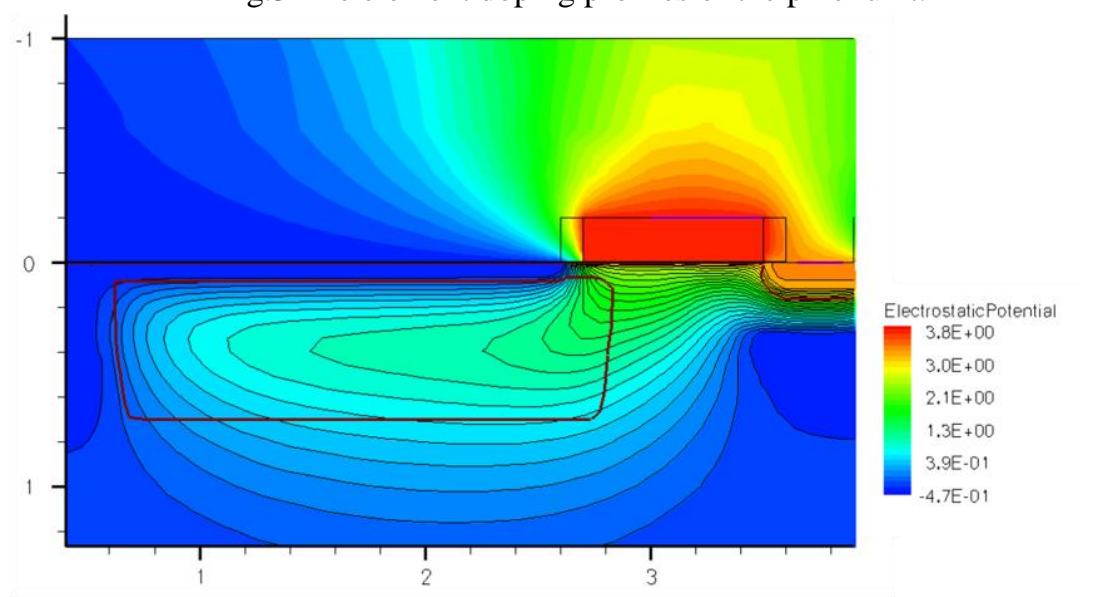

Fig. 4 Device simulation of charge transfers the PPD to FD node when the TG is turned on.

To understand why image lag increases with increasing TID, the potential of the charge transfer path of the pixel cell is analyzed by simulation. Fig. 5 shows the potential difference of the charge transfer path pre- and post-irradiation. In the pixel design, when the TG is turned on, there is a potential difference between the FD node and the PPD region, which acts as a driving force for charge transfer process. And the larger the potential difference, the faster the charge transfer speed. From Fig.5, it can be seen that there is a potential pocket (a potential pocket may exist if the TG overlaps too much the photodiode [9]) near the TG region, and the existence of the potential pocket leads to the generation of image lag at the macro-phenomenon. Fig. 6 presents the simulated potential change with different accumulated doses. In Fig. 6, we can see that the potential difference between the FD node and the 
PPD region is larger after irradiation, indicating that the charge transfer speed increases with the TID. However, a large potential difference (relative to the irradiation) occurs between the PPD region and the TG region, which leads to increasing the depth of potential pockets so that the charge cannot cross the potential pocket to FD node.

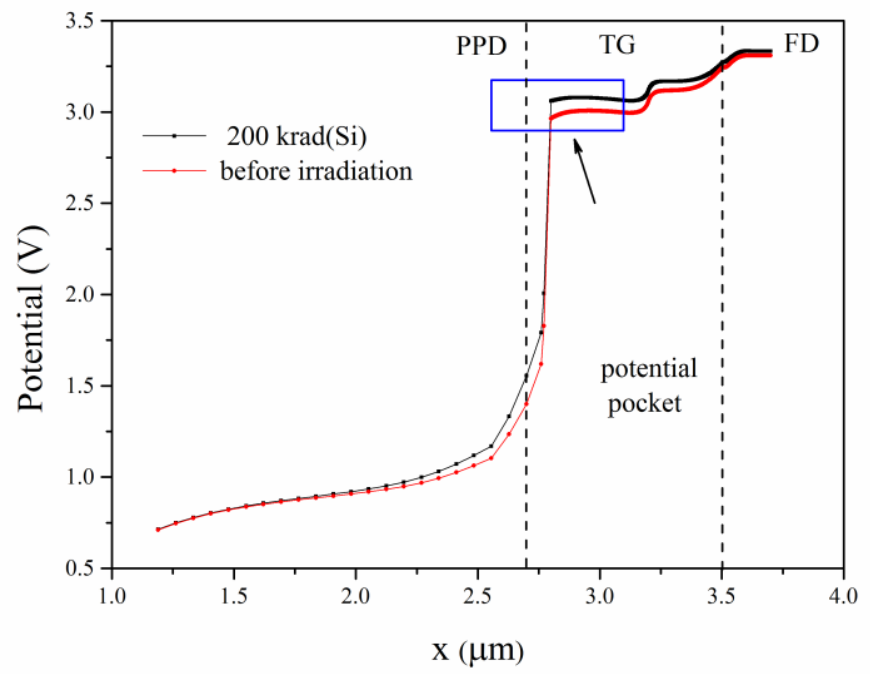

Fig. 5 Potential distribution on the charge transfer path pre- and post-irradiation.

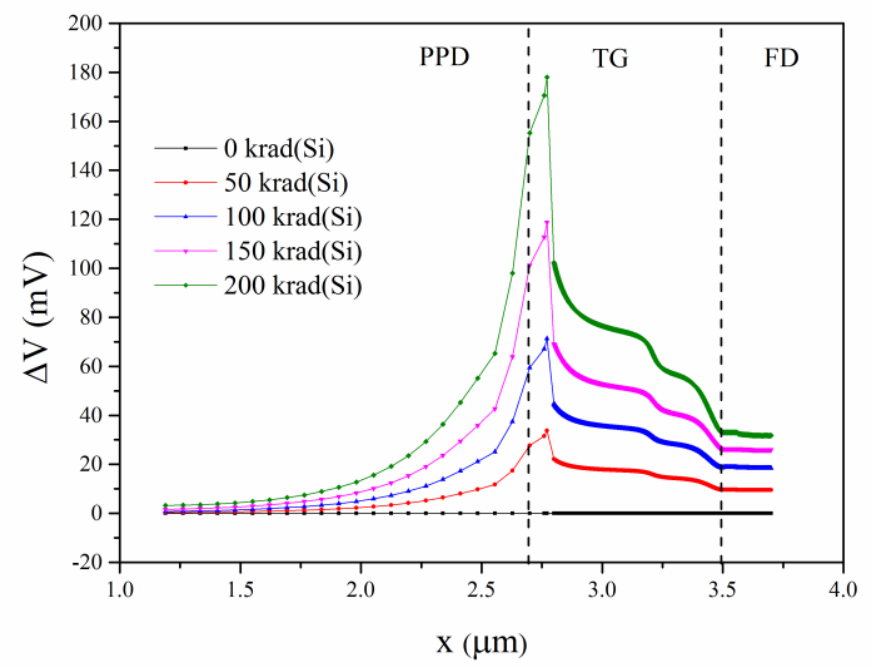

Fig. 6 Distribution of potential difference on the charge transfer path pre- and post- irradiation.

In this TCAD model, the defect model about trapped charge is introduced in the oxide layer. As a result, a large amount of the positive charges trapped is generated in the oxide layer after irradiation. The positive charges trapped will affect the entire electric field intensity distribution of the pixel model. It will also change the potential difference of the charge transfer path from the PPD to the FD node.

Fig. 7 is a schematic representation of the potential pre- and post- irradiation. In Fig.7, it can be seen that a large potential magnitude exists in the PMD region after irradiation. We can infer that the high potential caused by irradiation is one of the possible causes of the incomplete transfer of charge. 


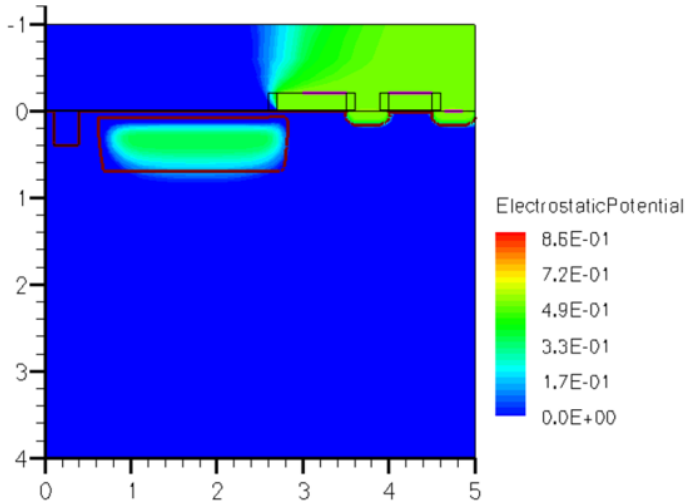

(a)

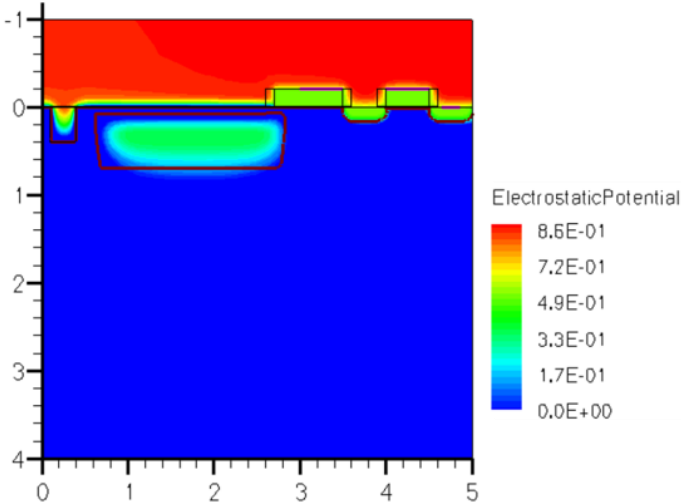

(b)

Fig. 7 Device simulation of the distribution of potential: (a) before irradiation; (b) after irradiation.

Fig. 8 shows the distribution of the positive charges trapped schematic when the TID reached $200 \mathrm{krad}(\mathrm{Si})$ in the simulation (the equivalent dose in the real experiment may differ as discussed at the beginning of the section). The positive charges trapped distribution will change the potential distribution in the oxide region. The potential distribution in the oxide region will affect the potential distribution in the bulk silicon, resulting in the signal charge unable to be successfully transferred to the FD node.

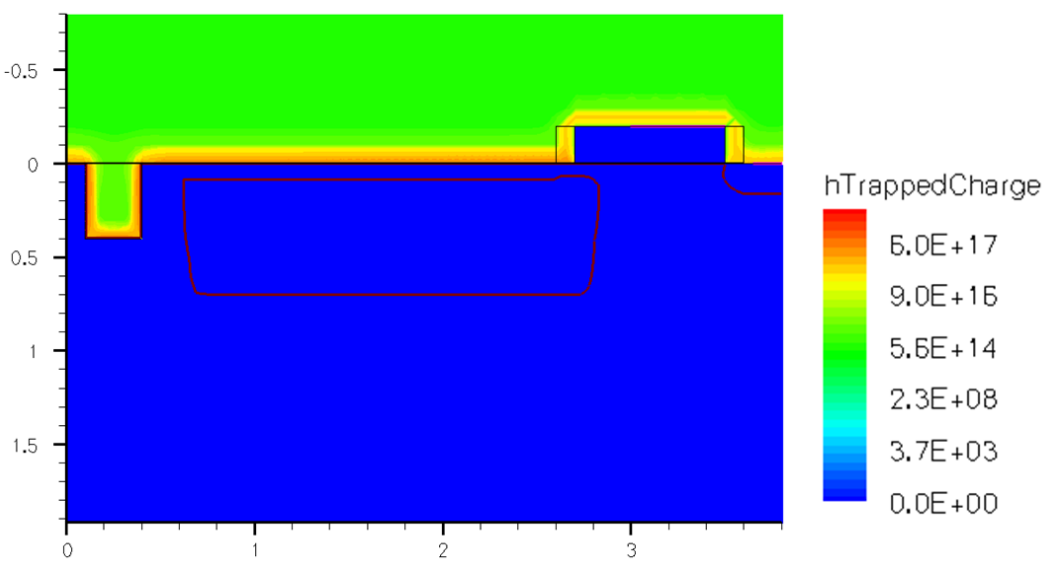

Fig. 8 When the TID reached $200 \mathrm{krad}(\mathrm{Si})$, the distribution of the positive charges trapped schematic.

\section{Conclusion}

In this paper, With the TID radiation test and the TCAD simulation of the 4T PPD CIS based on the standard $0.18 \mu \mathrm{m}$ CMOS technology, the degradation of the image lag problem has been investigated. With the TID increases, the degradation of the image lag get more and more serious.

In the TCAD simulation, the defect model about trapped positive charge is introduced in the oxide layer. By observing the distribution of the potential changes in the charge transfer path pre- and post-irradiation, potential change is significant in the junction of the PPD region and TG region after irradiation which leads to the expansion of the potential pocket in the junction impede the transfer of charge. By observing the distributions of potential and the positive charges trapped, we can confirm that a large number of positive charges trapped caused by irradiation are one of the factors causing the incomplete transfer of charge.

It is well known that the main cause of image lag in 4T PPD CISs is the potential barrier or pocket between the PPD region and the TG region. Radiation induced positive charges trapped in the dielectrics around this overlap region (e.g., the PMD and the spacers) lead to an additional electric filed that trends to lower potential barriers and increase the depth of potential pockets [8-9]. However, in this paper, the representativeness and accuracy of the presented simulations are limited by several factors: a) We have not considered the case where a potential barrier exists in the charge transfer path. b) In this TCAD simulation model, some structures are simplified, such as the main material of the spacer region which is constituted only by $\mathrm{SiO}_{2}$ in this simulation. c) The physical simulation model of TID radiation is not comprehensive enough. For example, interface states were not considered whereas they might play a role in the final degradation [8-12]. Nevertheless, the simulation is still valuable to analyze and understand the qualitative effects though the absolute magnitude of the simulated degradation may not be accurate.

In the future, more radiation experiments of the different types of 4T PPD CIS and optimization of TID radiation model will be 
carried out to confirm the conclusion of this paper.

\section{Acknowledgments}

This work was supported by the National Science Foundation of China (Grant Nos.11305126, 11235008) and the Foundation of State Key Laboratory of China (Grant No. SKLIPR1211).

\section{References}

[1] R.Turchetta, Nucl. Instrum. Methods A 458(2001) 677.

[2] V.Goiffon, P.Magnan, O.saint-Pe, F.Bernard, G.Rolland, Nucl. Instrum. Methods A 610(2009) 225.

[3] V.Goiffon, C.Virmontios, P.Magnan, P.Cervantes, F.Corbiere, M.Estribeau, P.Pinel, Proc. of SPIE 7826(2010) 78261 S-1.

[4] D.A.Evans, P.P.Allport, G.Casse, A.R.Faruqi, B.Gallop, R.Henderson, M.Prydderch, R.Turchetta, M.tyndel, J.Velthuis, G.Villani, N.Waltham, Nucl. Instrum. Methods A 546(2005) 281.

[5] J.Tan, (Ph.D.dissertation), Technische Universiteit Delft, The Netherlands, 2013.

[6] S.Place, J.-P.Carrere, S.Allegret, P.Magnan, V.Goiffon, F.Roy, IEEE Trans. Nucl. NS 59(2012) 909.

[7] I Inoue, H. Nozaki, H.Yamashita, T.Yamaguchi. IEDM. 1999:883-886.

[8] Z.X.Cao, Q.L.Li, Y.H, Q.Qin, P.Feng, L.Y.Liu, N.J.Wu, Journal of Simiconductors. 35(2014) 114010-1.

[9] V.Goiffon, M.Estribeau, O.Marcelot, P.Cervantes, P.Magnan, M.Gaillardin, C.Virmontois, P.Martin-Gonthier, R.Molina, F.Corbiere, S.Girard, P.Paillet, C.marcandella, IEEE Trans. Nucl. NS 59(2012) 2878.

[10] V.Goiffon, M.Estribeau, P.Cervantes, R.Molina, M.Gaillardin, P.Magnan, IEEE Trans. Nucl. NS 61(2014) 3290.

[11] Z.J.Wang, Y.W.Ma, J.Liu, Y.Xue, B.P.He, Z.B.Yao, S.Y.Huang, M.Liu, J.K.Sheng, Nucl. Instrum. Methods A 820(2016) 89-94.

[12] T.P.Ma, P.V.Dressendorfer. Ionizing radiation effects in MOS devices and circuits. Wiley. New York. 1989.

[13] J.-L. Leray. Total Dose Effects: Modeling For Present And Future. IEEE NSREC Short Course, 1999. 